EOMmUn: Communication et organisation

OHG

Communication et hôpital - Analyse et diagnostic

\title{
Conférence - Les réseaux de soins
}

\section{Pascal Forcioli}

\section{OpenEdition}

Journals

Édition électronique

URL : http://journals.openedition.org/communicationorganisation/2992

DOI : 10.4000/communicationorganisation.2992

ISSN : 1775-3546

\section{Éditeur}

Presses universitaires de Bordeaux

\section{Édition imprimée}

Date de publication : 1 mai 1994

ISSN : 1168-5549

\section{Référence électronique}

Pascal Forcioli, «Conférence - Les réseaux de soins », Communication et organisation [En ligne], HS N 1 | 1994, mis en ligne le 27 mars 2012, consulté le 19 avril 2019. URL : http://journals.openedition.org/ communicationorganisation/2992 ; DOI : 10.4000/communicationorganisation.2992

Ce document a été généré automatiquement le 19 avril 2019

(c) Presses universitaires de Bordeaux 


\title{
Conférence - Les réseaux de soins
}

\author{
Pascal Forcioli
}

1 Le thème que je vais traiter devant vous porte sur « la communication dans les réseaux de soins » Ce thème a fait l'objet d'un groupe de travail à l'initiative de la Direction des Hôpitaux en 1992-1993 et d'une publication dans Informations Hospitalières. Le groupe de travail, que j'ai eu l'honneur d'animer, était composé de chargés de communication hospitaliers, de directeurs d'hôpitaux et de médecins hospitaliers.

2 Les différents participants avaient des expériences diverses de réseaux de soins hospitaliers : soit pour des malades chroniques, soit pour des malades atteints du sida ou plutôt malheureusement dans des phases terminales, soit des expériences de réseaux en matière de gériatrie et gérontologie selon l'optique que l'on a de ce type de réseau.

3 Ce qu'on peut dire pour commencer, c'est que les réseaux de soin sont un concept relativement nouveau dans le monde de la santé et ne concernent pas seulement les hôpitaux mais tous les partenaires du système de santé.

Les réseaux de soin font l'objet de définitions récentes sur lesquelles on va revenir tout à l'heure, avec des terminologies que l'on retrouve notamment dans le domaine du sida, mais aussi maintenant dans le domaine de la toxicomanie ou de l'alcoologie et puis bien entendu, aussi dans le domaine de la gérontologie.

5 Le réseau de soin est une réponse originale à la prise de conscience par tout le secteur santé de notre société, de la nécessité de prendre en charge globalement le patient. C'està-dire en tant que personne humaine, en tant que personne sociale, en tant que personne malade, en tant que personne ayant un affect, une histoire.

6 L'idée du réseau est d'essayer de mobiliser tous les partenaires qui peuvent aider à la réinsertion du patient ou à son retour le plus rapide au domicile et à sa reprise de la vie sociale. Ça veut dire autant les partenaires hospitaliers que les partenaires extrahospitaliers que des partenaires médicaux et infirmiers et autres.

7 On constate que c'est surtout l'apparition du sida qui a été en matière de santé publique, l'occasion de développer la coordination des acteurs sous la forme de réseau ville-hôpital. Ce terme de réseau ville-hôpital indique bien la fluidité, je dirais de la filière de soin entre la ville et l'hôpital. 
8 L'idée constitutive du réseau, c'est que le patient puisse être pris en charge tantôt par la ville tantôt par l'hôpital avec des soins gradués et coordonnés.

Ce sont les deux déterminants que l'on va retrouver dans des réseaux : la graduation des soins et des services rendus et la coordination des actions des différents partenaires, «le soin » étant entendu au sens le plus large du terme.

Permettez-moi de citer quelques définitions dont une que j'ai relevée dans une circulaire du 4 juin 1991 du Ministère de la santé, Direction des Hôpitaux, Direction Générale de la Santé: "le réseau de soin est une organisation locale ouverte. Cette organisation est centrée sur un site hospitalier parfois sur plusieurs. Il doit conserver une taille suffisamment petite pour favoriser une connaissance mutuelle entre les professionnels, et pour permettre aussi d'assurer le suivi commun des patients. Il doit être aussi suffisamment ouvert pour fédérer sur un site donné des pratiques de collaboration qui sont restées parallèles jusqu'à maintenant. Ne pouvant être réservé à une profession particulière, il doit demeurer accessible aux professionnels qui concourent à la prise en charge globale des patients. Toutefois bien entendu, les liaisons entre la médecine de ville et la médecine hospitalière y occupe la place centrale ».

11 Je vous propose ensuite la définition qui a été celle retenue par le groupe de travail organisé par la Direction des hôpitaux en 1992-1993 : « un réseau de soin est un ensemble organisé, on pourrait même dire finalisé, de plusieurs personnes physiques ou morales » Ces personnes peuvent être des médecins qui agissent en tant que médecins libéraux, des cabinets médicaux, des sociétés civiles et des moyens, des établissements privés et publics de santé, des associations...

Ces personnes physiques et morales constituent les acteurs du réseau.

13 À l'origine, les acteurs sont dispersés dans une zone territoriale donnée. Ça veut dire qu'avant l'existence du réseau, ils travaillent chacun dans leur coin, et le réseau c'est la mise en communauté de ces différents acteurs et la mise en communication de ces différents acteurs. Ces acteurs ont des compétences différentes, mais des compétences complémentaires et ils agissent dans un objectif commun : la prise en charge globale du patient. Ceci veut dire soit le soin, soit la réinsertion, soit lutter contre la conséquence de la maladie et en retarder ou diminuer les effets.

14 Ce qui est très important aussi dans notre définition, qu'on a voulu assez complète, c'est qu'on estime que «les acteurs du réseau doivent agir dans un objectif commun, mais aussi selon des normes et des valeurs partagées ", et ça c'est très important en termes de communication. Car la communication, c'est aussi dégager les normes et les valeurs que l'on va choisir de partager et auxquelles on va adhérer.

La communication pourra se faire notamment sur ces valeurs.

16 Bien entendu, tout ça repose sur une base de volontariat, on pourrait même dire, de confiance, entre les acteurs.

17 «L'objectif ultime, c'est améliorer la prise en charge globale d'une communauté de malade ».

18 J'ai relevé dans une circulaire encore plus récente que celle que j'ai citée tout à l'heure, datée du 7 mars 1994, et relative au réseau ville-hôpital dans le cadre de la prise en charge des toxicomanes: "un réseau est une organisation ouverte qui s'appuie au minimum sur un site hospitalier, sur un regroupement de médecins généralistes déjà 
impliqués dans la prise en charge des toxicomanes et sur un centre spécialité de soins aux toxicomanes lorsqu'ils existent ».

19 Ce qu'il faut retenir de ces différentes définitions, c'est la communication entre les différents acteurs, leur complémentarité et le fait aussi que les acteurs dans le réseau ne fonctionnent pas sur un principe d'autorité, mais sur le principe de la coordination. Chacun est autonome dans son action, chacun est responsable au plan déontologique, et au plan pénal de son action, mais ces actions sont complémentaires, sans rapport hiérarchique entre les différents éléments du réseau.

20 Bien entendu, le réseau est un lieu d'échange, de circulation de l'information autour du malade. C'est la communication sur le patient, organisée autour du dossier du patient, commun et partagé entre les différents acteurs. Quand je dis partagé cela ne veut pas dire que toute l'information est accessible à tout le monde car bien entendu, il convient de respecter les règles de déontologie, et le secret professionnel.

21 Les structures en réseau, répondent à une évolution des systèmes hospitaliers vers plus de modernité mais aussi vers plus d'adaptabilité, de flexibilité, mais aussi surtout plus d'humanité parce que c'est ça qui compte avant tout.

22 Les acteurs dans un réseau sont multiples. Selon l'objet du réseau, on va choisir des partenaires différents, ça peut aller des hôpitaux publics aux organismes de sécurité sociale, en passant par les associations, les compagnies d'assurances, les structures mutualistes, les services d'urgence, les acteurs institutionnels ou financiers etc.

23 Ce qui est essentiel aussi dans le réseau, c'est le fait que le réseau fonctionne de manière coordonnée et pour qu'il fonctionne de manière coordonnée, il faut qu'il y ait un coordonnateur qui sera l'élément pivot de la communication dans le réseau. Vous pourrez observer différents types de réseaux qui existent dans le domaine des soins. Vous observerez toujours l'existence d'un, deux ou plusieurs coordonnateurs.

Le financement du réseau ou des actions des acteurs dans le réseau peut être multiple. Par exemple dans un réseau de prise en charge des malades, vous avez des actions sanitaires, mais vous avez aussi des actions sociales et ce ne sont pas les mêmes financements. Les actions sanitaires font intervenir le financement de l'assurance maladie, les actions sociales font intervenir d'autres financements, soit des financements associatifs, soit des financements des conseils généraux, soit des financements directs par les malades payants le service.

On observe généralement une double structure dans les réseaux: une structure horizontale qui est coordonnatrice et des structures verticales. Ces structures verticales, ce sont les structures des différents intervenants, qui sont spécialisées. Le coordonnateur, c'est le lien entre les structures verticales.

Par exemple, j'ai retenu la structure de l'association qui s'occupe de l'assistance régionale aux insuffisants respiratoires des Pays de Loire, et qui a pour nom ARIRPLO. Cette association a une structure horizontale coordonnatrice qui se compose d'une cellule technique avec un technicien. Pourquoi ? Parce que cette association met à disposition des malades insuffisants respiratoires, des matériels d'assistance respiratoire et de ventilation, et que le problème essentiel pour les malades qui ont ce matériel à domicile et qui vont devoir s'en servir dans des moments difficiles, (ce qui va éviter leur hospitalisation en urgence), c'est la connaissance, la maîtrise de l'équipement, du matériel et donc de la technique. 
27 Le rôle de coordination du technicien va très loin parce que non seulement il installe la machine, il explique au patient et aussi à sa famille comment on s'en sert. Mais également il est le relais entre le domicile et la structure hospitalière ou les autres partenaires médicaux qui interviennent dans l'association ARIRPLO. Il lui arrive de signaler des problèmes aux équipes hospitalières et régulièrement d'ailleurs, il est intégré dans le réseau. Régulièrement le réseau se réunit en réunion, associant les différents partenaires professionnels et à cette occasion les partenaires professionnels échangent et le technicien peut donner des informations sur la façon dont le patient vit son insuffisance respiratoire au domicile. Vous voyez ce rôle de coordination, d'information est tout à fait fondamental. il contribue au renforcement de l'autonomie du patient et le cas échéant à son éducation sanitaire et technique.

J'aurais pu prendre un autre exemple qui est celui de la Coordination Sanitaire et Sociale que je connais dans le sud de L'Oise, qui a d'abord été expérimentée sur un canton correspondant à une zone de population de 70000 habitants, sur les cantons de Senlis et Chantilly. Elle est aujourd'hui étendue à l'ensemble d'un secteur sanitaire, c'est-à-dire une subdivision de la région Picardie correspondant à tout le sud du département de L'Oise, soit une population, de 310000 habitants.

Cet organisme est une fédération d'associations localisées dans plusieurs villes. Son objectif est de constituer un réseau de services et de soins gradués coordonnés avec le système hospitalier destiné à favoriser le retour des patients au domicile le plus rapidement possible, voire d'éviter les hospitalisations. Son action consiste à mettre à disposition au domicile des services de soins, des services médicaux, des services hôteliers et ménagers, des services d'assistance aux personnes malades et aux personnes, qui ont perdu leur autonomie de vie. Cet organisme repose sur une coordination infirmière forte. Les actions au domicile sont assurées par les libéraux (médecins, infirmières, kinésithérapeutes). Ces libéraux sont rémunérés à l'acte par la Coordination qui sert de tiers-payant et qui est remboursée par l'assurance maladie sur la base d'un forfait soins.

L'infirmière coordinatrice est le pivot du système. Elle assure l'expertise des besoins du malade à son domicile et organise les moyens (soins et services) nécessaires compte tenu du malade. Elle est la base de la communication interne au réseau.

31 Le réseau de soins, on l'a dit, c'est un lieu de communication. La communication est nécessaire au réseau. Le réseau est communication par lui-même. Et puis on est en train de découvrir dans les réseaux hospitaliers, dans les hôpitaux, la nécessité de la communication externe. Car le réseau, c'est une organisation, ça veut dire que cette organisation a besoin de se faire connaître, de se faire reconnaître vis-à-vis de ses partenaires financiers, mais aussi vis-à-vis des acteurs potentiels, des clients potentiels. Le réseau doit faire reconnaître son utilité sociale et sanitaire. Différents moyens peuvent être employés, soit en engageant des actions de communication vers des institutions sanitaires, des institutions sociales, des collectivités locales, mais également auprès des comités et revues scientifiques, auprès d'associations de médecins...

Parmi les différentes formes de communication interne, on retrouve les formes classiques de communication qu'on a dans n'importe quelle organisation : d'abord des réunions. il faut en effet que les gens du réseau qui sont dispersés comme on l'a dit, se rencontrent pour coordonner leurs actions, pour aussi évaluer ensemble l'impact de leurs actions par rapport à un patient donné. Il faut qu'ils aient des échanges sur leurs pratiques respectives pour enrichir ces pratiques. Certains réseaux mettent en place des 
commissions de travail, des commissions spécialisées, des commissions permanentes; certains réseaux sont regroupés en fédération, auquel cas l'association qui est elle-même un réseau s'intègre dans un réseau encore plus grand, comme dans l'exemple de la Fédération des Associations de Coordination Sanitaire et Sociale de l'Oise, mais aussi dans l'exemple de l'ANTADIR, qui est une association nationale qui regroupe les associations s'occupant des insuffisants respiratoires dans les régions.

L'élément essentiel de la communication dans le réseau, c'est le dossier commun du patient. Ce dossier est un dossier à la fois médical infirmier, social et psychologique, toutes facettes qui sont nécessaires à la prise en charge du patient. Ce dossier peut être informatisé, ou manuel. Il faut savoir que si c'est un dossier informatisé, il ne faut pas oublier d'être en conformité avec les recommandations sur la loi «informatique et libertés ", et il faut donc avoir un avis favorable pour la mise en œuvre d'un traitement automatisé et nominatif d'informations, sous forme d'autorisation de la C.N.I.L. Ce dossier va être véritablement le point nodal de la communication du réseau de soins.

Le réseau peut également avoir des fichiers et ces fichiers sont très utiles : l'hôpital Bichat réalise un fichier sur les services dont il dispose. Il faut que le réseau ait lui-même un fichier sur son organisation qu'il puisse distribuer à l'extérieur. Mais le réseau aussi est un lieu où l'on va trouver des fichiers sur les correspondants qui sont utiles dans le cadre de la prise en charge des patients; par exemple dans le cadre d'un réseau SIDA, il est intéressant de savoir quels sont les dentistes, les infirmiers, les différents professionnels qui acceptent de s'occuper des malades sidéens, parce que vous savez que tous les professionnels ne l'acceptent pas forcément. Donc le réseau aura une mission d'information : il doit pouvoir être sollicité par les usagers ou par les acteurs internes ou externes pour mettre en relation le patient avec le bon correspondant; il doit pouvoir fournir des coordonnées particulières, par exemple dire au malade bénéficiaire du réseau, quels sont les fournisseurs qui peuvent apporter du linge ou des repas à domicile.

Outils également traditionnels de communication, le Minitel, et le journal du réseau. Je citerai par exemple, le journal "Bulle d'air " à Angoulême. "Bulle d'air", ça fait référence à deux éléments: l'air, dont on a besoin pour respirer et qui est particulièrement indispensable aux insuffisants respiratoires (comme à chacun sauf que la capacité d'absorption du traitement de cet air n'est pas forcément la même), et puis «bulle », ABD est une association d'Angoulême, cité de la bande dessinée et du festival annuel de la B.D.

J'insisterai ici sur les objectifs de la création d'un réseau. Pourquoi créer un réseau?

Pour assurer une prise en charge globale des patients, pour renforcer les relations entre la médecine de ville et la médecine hospitalière ; c'est un objectif, non seulement de santé publique, mais aussi d'économie de la santé : favoriser le maintien à domicile, humaniser les rapports entre le système de soins et ses utilisateurs en rapprochant les soins $d u$ domicile, améliorer l'accès aux soins pour tous les malades, en particulier pour les plus démunis, évaluer l'amélioration de la qualité des soins. Pour des professionnels, ça peut être, par exemple, se sécuriser, parce que quand on fait partie d'un réseau on bénéficie du travail de spécialistes, de l'apport d'experts compétents sur un problème difficile et complexe (et les problèmes de santé sont de plus en plus difficiles et complexes à traiter, et un médecin généraliste a parfois du mal à les résoudre seul).

38 Je voudrais insister sur deux derniers éléments dans cet exposé, qui retrace donc assez brièvement les travaux que nous avons conduits l'an passé : c'est, d'une part, l'aspect de 
communication de l'acte de naissance du réseau et, d'autre part, les aspects de communication externe.

Un réseau, c'est souvent quelque chose de nouveau qu'on met en place. Soit l'hôpital y participe, soit l'hôpital n'y participe pas. Et là encore on observe souvent que les hôpitaux sont impliqués dans la création de ces réseaux : certains de ces hôpitaux bénéficiant d'une cellule de communication, le chargé de communication hospitalière, tout naturellement, trouve là un terrain de travail. Car s'il doit faire de la communication pour son hôpital, dès lors que son hôpital participera à un réseau, il lui faut aussi participer à la communication du réseau. Un moment essentiel, c'est la naissance, le moment où on va porter puis baptiser puis élever le réseau. La communication externe et de la communication interne, sur cet événement sont nécessaires. Communication externe, auprès de qui ? Auprès des partenaires potentiels.

On part de la naissance (on est un petit nombre pour une naissance). Il faut intéresser les partenaires potentiels en tant que participants à la filière de soins. Puis il y a les patients qui vont être la cible également du réseau, et puis les financeurs ou les tuteurs dans le cas où le réseau est soumis à un contrôle de légalité ce qui existe assez fréquemment. Le message à faire passer auprès des cibles sera centré autour des objectifs du réseau. On dira : pourquoi on met en place le réseau, pour quelle mission, pour quels patients, pour quelles actions. On pourra moduler ce message en fonction des cibles vers lesquelles on destinera le message ; ça veut dire qu'on ne peut pas donner le même message aux futurs acteurs du réseau, aux professionnels et aux malades. Choisir ensuite un certain nombre de moyens et réserver des moyens, ça veut dire prévoir aussi dans le réseau un budget de communication pour éviter d'être en situation de faire «la manche ». Donc des moyens, une politique d'image, des adresses, des rencontres avec des partenaires potentiels, des participations à des salons, différents forums et puis des dossiers de presse. Une politique d'image ça peut passer par le choix d'un logo. Quand on voit le logo on dit, ça c'est le logo de tel réseau ; on a une charte graphique, un outil classique de communication, on édite des plaquettes de présentation pour faire émerger l'image de ce futur réseau, des adressages dont les messages seront bien ciblés...

41 Il y a aussi la communication interne : entre ceux qui lancent le réseau, entre ceux qui vont rejoindre le réseau, entre les clients aussi qui vont être bénéficiaires du réseau. Ce sera très important parce que bien entendu, au sein de chaque organisation - et le réseau, on l'a dit, c'est une organisation - il est nécessaire d'avoir une communication interne pour dynamiser les gens, pour les faire échanger, pour faire évoluer leurs pratiques, pour qu'ils se sentent aussi appartenir à un ensemble commun. Ca pourra être un journal interne,... Si on fait partie de n'importe quelle association, sportive, culturelle, on reçoit toujours de la documentation, un petit journal, un petit bulletin, annuel ou trimestriel et le réseau ne doit pas échapper à cela, je dirai même qu'il doit avoir une communication de type vraiment professionnel.

42 La communication externe est souvent oubliée, mais il faut absolument y penser pour satisfaire deux objectifs : la promotion du réseau et sa pérennité. C'est très important. Trois problèmes sont rencontrés pour pérenniser le réseau : il faut trouver de l'argent, des professionnels et aussi des clients, c'est-à-dire soit les conserver, soit étendre la population qui est bénéficiaire du réseau car l'objectif reste, ne l'oublions pas, d'offrir un service de santé et d'offrir le meilleur service de santé pour le plus grand nombre. Ca peut passer par la recherche de fonds, par des actions promotionnelles que vous connaissez bien, de publication, de formation, de colloques, d'actions d'éducation, d'actions de 
prévention, là encore on le redit, de parutions, de publications, de notes, de bulletins, de journaux et puis aussi ne pas oublier des relations avec les médias. C'est-à-dire que le réseau ne doit pas se contenter d'avoir de temps en temps des articles dans la presse, mais aussi maintenir de manière relativement étroite des relations constantes avec elle; et l'informer régulièrement des événements dont il assure l'organisation et qui sont susceptibles d'intéresser le public et les usagers actuels et potentiels.

Pour conclure, on pourrait dire que c'est une façon nouvelle de travailler. Travailler en réseau cela veut dire travailler ensemble, dans une structure, mais cela veut dire aussi travailler de manière ouverte avec l'extérieur, avec l'environnement et cela veut bien dire communiquer en interne autant qu'en externe. C'est très important cette double communication. Merci de votre attention.

\section{QUESTIONS} Hôpitaux au Ministère de la santé de provoquer une sorte de colloque sur les réseaux de soins en France, et puis d'essayer à partir de là, de déterminer le nombre de réseaux. Je crois que ce serait une entreprise d'abord difficile à organiser, ce qui explique qu'on ne l'ait pas fait; et d'autre part, il y a vraiment une multiplicité de réseaux. On a parlé des réseaux ville-hôpital-SIDA ça peut être relativement bien recensé ; il y en a pas mal qui fonctionnent sur Paris, il y a des réseaux d'arrondissements, autour de centres hospitaliers, en Province. Vous commencez à avoir des réseaux au niveau de la gériatrie. On pourrait dire aussi que la psychiatrie est depuis longue date organisée en réseaux sur le concept de ce que l'on appelle la sectorisation psychiatrique. Il y a également des domaines nouveaux qui sont concernés par l'apparition des réseaux : par exemple pour la prise en charge des malades atteints du cancer. Donc là on a besoin de mobiliser des partenaires différents ; le principe est toujours à peu près le même, on crée un réseau dès lors qu'on a besoin d'associer dans un même travail pour un même objectif de soin, les professionnels de différents secteurs, d'environnements différents, des professionnels de villes et des professionnels hospitaliers par exemple, ou des médecins et des infirmiers ou des médecins et des infirmiers et des techniciens. Mais je ne sais pas répondre précisément à votre question.

47 M. BADENES : Ma question rejoignait en fait un peu celle qui vient d'être posée. Vous dites qu'apparemment, c'est difficile d'avoir une liste et qu'il n'y a pas d'annuaire qui existe. Comment faire?

M. FORCIOLI : Je pense que dans un département, dès lors qu'il s'agit de réseaux de soins, les services préfectoraux dont la DDASS, doivent avoir l'information sur l'organisation des réseaux de soins existants, ou les services de l'assurance maladie; les uns ou les autres peuvent être des correspondants qui peuvent être interrogés sur l'existence ou non des réseaux et sur l'objet de ces réseaux dans le département.

Mme CARAYOL : Concernant la structure juridique de ces réseaux, est-ce qu'il y a une forme qui a été adoptée par une majorité d'entre eux? 
50 M. FORCIOLI : Oui, absolument, il y a une forme très répandue, c'est la forme associative, relevant de la loi de 1901. Maintenant, il y a des formes un peu plus sophistiquées qui peuvent être offertes, notamment grâce à l'évolution des textes régissant l'organisation hospitalière, (loi du 31 juillet 1991) telles que les groupements d'intérêt économique ou des groupes d'intérêt public. Mais la formule associative est la formule la plus souple. Elle offre un premier avantage, c'est que le réseau acquiert son autonomie. L'association confère à l'organisme la personnalité morale, avec un personnel propre, un financement propre, des règles d'organisation propres, constituées par le règlement intérieur de l'association. L'administration est propre : c'est le conseil d'administration, l'exécutif, le bureau. Donc tous les éléments sont là, pour que le réseau puisse fonctionner, et n'importe quelle personne morale ou personne physique peut adhérer, je dirais, sans conditions particulières à une association. C'est vraiment la formule la plus répandue. Dans les participants au groupe de travail l'an passé, il y avait des gens qui avaient des expériences de réseaux différentes, le point commun que nous avions tous, c'était que ces réseaux dans lesquels nous étions étaient des associations.

Mme CARAYOL : Vous parliez tout à l'heure des problèmes de financement. Quand vous avez à trouver des partenaires financiers, c'est pour financer le réseau dans quelles proportions ? Est-ce que l'hôpital est le premier financeur?

52 M.FOKCIOLI : Je pense que ça dépend de l'objet du réseau. Je vais prendre l'exemple du réseau de soins et de services de la Coordination Sanitaire et Sociale sur le Sud de l'Oise, c'est-à-dire une population de 300000 habitants. Cette fédération d'associations a pour objet, de promouvoir, d'organiser le maintien à domicile des personnes soit malades, soit dépendantes (ça ne vise pas uniquement les personnes âgées, ça vise également les malades chroniques ou les malades en fin de vie). Ce réseau particulier a une double mission : il délivre à la fois des soins et il permet aux personnes de rester à leur domicile. C'est-à-dire qu'il leur fournit des aides pour rester à leur domicile et donc là, il y a un double financement qui est organisé et qui est propre à ce réseau de soins, qui est, d'une part, le financement assurance-maladie pour le soin, avec un forfait ou plutôt plusieurs forfaits établis selon la graduation des soins, déterminés par convention chaque année entre l'assurance-maladie, (caisse régionale d'assurance-maladie et caisse primaire concernée), et la Fédération des associations de coordination sanitaire et sociale; et d'autre part, il y a un forfait, je dirais, hôtelier, (aide ménagère, aide de vie) là encore gradué selon les services qui sont rendus. Et ce forfait service est payé soit directement par les bénéficiaires ou leur famille si ces personnes-là peuvent payer, soit pris en charge par le département au titre de l'aide sociale.

53 M. BADENES : Vous avez parlé de normes et de valeurs partagées, de volontariat, de confiance; concrètement, au départ justement de la création du réseau, l'impulsion initiale, elle part de l'affinité, de collaborations professionnelles. Comment ça se structure concrètement? ils se décident, et ils créent une association et ils essayent de trouver de nouveaux partenaires...vous parliez de deux personnes au départ?

M. FORCIOLI : Absolument, il suffit de deux personnes au départ pour que naisse une première volonté commune. li faut qu'il y aient des partenaires qui décident de travailler ensemble, qui y trouvent un intérêt : ça peut être deux ou trois personnes, enfin quand je dis " personnes ", ce sont des personnes physiques ou des personnes morales, ça peut être l'hôpital qui, autour de lui, va fédérer un réseau de soins ; par exemple, dans le cas de la Coordination Sanitaire et Sociale, l'initiative est revenue à un chef de service hospitalier, responsable du moyen séjour/rééducation au Centre Hospitalier de Senlis. Que fait ce chef 
de service dans son service? il cherche à réadapter des gens, des malades, pour qu'ils retournent à leur domicile; et il était confronté au problème de durées de séjour relativement longues, et d'autre part, à la pression des services de médecine, de chirurgie de l'établissement et des autres établissements de la région, qui souhaitaient lui adresser des patients. Donc, il a envisagé dans un premier temps, le réseau de soins à domicile comme un relais entre l'hôpital et le domicile pour permettre ce retour le plus rapide, pour dégager des lits d'hospitalisation en moyen séjour, et pour faire en sorte que son service réponde mieux à la pression des services actifs de médecine et de chirurgie. Donc là, l'initiative est partie de ce médecin hospitalier. il a trouvé des partenaires qui étaient prêts au niveau médical à rentrer dans un cadre associatif. Il a ensuite trouvé des partenaires infirmiers qui acceptaient de travailler dans un cadre associatif. Le premier travail de ce chef de service a été de convaincre de l'utilité de ce réseau, de ce concept de réseau, les partenaires financiers c'est-à-dire le Conseil Général et l'assurance-maladie. Il faut bien avouer que ce chef de service a un atout considérable pour convaincre l'un des partenaires financiers puisqu'il est vice-président du Conseil Général, ça aide. Mais par contre, s'il a su bien convaincre le Conseil Général, il lui a fallu ensuite convaincre l'assurance-maladie. Et le problème qui se pose aujourd'hui avec l'extension de son dispositif alternatif dans le département, c'est qu'il concurrence les structures de maintien à domicile qui préexistaient aux associations de coordination. Et tout l'enjeu consiste aujourd'hui à faire entrer les associations de maintien à domicile, comme les médecins de ville, mais ça pose plus de problèmes que les médecins de ville, dans le partenariat du réseau. Donc là, voilà un exemple où on voit comment le réseau s'est mis en place, avec quels partenaires, mais aussi quelles difficultés il peut rencontrer.

Mme PSUIK : Je connais un réseau de soins coordonnés dans le Nord qui prend en charge des malades cancéreux. Ces malades sont alternativement à domicile et à l'hôpital. Nous avons des difficultés pour réaliser à domicile les mêmes protocoles de soins pour des raisons matérielles. Y a t-il une solution?

M. FORCIOLI : C'est justement là qu'intervient la réponse du type de réseau qui a été mis en place sur le Sud de l'Oise. Parce que ce réseau a un partenariat avec l'hôpital au niveau des protocoles de soins, au niveau de certains matériels. Par exemple, on a inclus dans les accords qui nous lient avec ce réseau, la possibilité pour le réseau d'appeler des consultants hospitaliers pour que ces consultants fassent des visites à domicile, non pas pour prescrire au domicile à la place du médecin traitant qui fait partie du réseau, mais pour soutenir, assister, conseiller le médecin traitant qui intervient dans le réseau. $\mathrm{Ca}$ veut dire que c'est quand même le médecin traitant qui prescrit et qui prend en charge sa responsabilité en tant que prescripteur. S'il y a besoin de matériel particulier, on sait les mettre à disposition et puis après il $\mathrm{y}$ a simplement des règlements financiers qui se font entre l'association et l'hôpital par le biais de conventions. Ca montre que c'est très très ouvert. Et l'objectif du réseau c'est bien qu'il y ait une perméabilité entre l'hôpital et le domicile et que le plus souvent le malade retourne ou reste à domicile et je dirais même, si possible, il faut qu'il meure au domicile, ce qui est très important pour lui comme pour sa famille en matière de travail de deuil comme on le sait quand on analyse ces questionslà. 


\section{AUTEUR}

PASCAL FORCIOLI

Directeur du CH de Senlis 